\title{
COMPRAS PÚBLICAS DA AGRICULTURA FAMILIAR PARA A ALIMENTAÇÃO ESCOLAR EM MUNICÍPIOS DO ESTADO DO RIO DE JANEIRO
}

\author{
Patrícia Camacho Dias ${ }^{1}$ \\ Kamilla Carla Bertu Soares ${ }^{2}$ \\ Daniele Mendonça Ferreira ${ }^{3}$ \\ Luiz Humberto da Silva ${ }^{4}$ \\ Daniele da Silva Bastos Soares ${ }^{5}$ \\ Patrícia Henriques ${ }^{6}$ \\ Silvia Eliza Almeida Pereira de Freitas ${ }^{7}$ \\ Luciene Burlandy Campos de Alcântara ${ }^{8}$ \\ Roseane Moreira Sampaio Barbosa ${ }^{9}$
}

Resumo: O objetivo do estudo foi analisar o perfil de compras da agricultura familiar para o Programa Nacional de Alimentação Escolar em municípios do Estado do Rio de Janeiro. Buscou-se compreender as características sociodemográficas dos municípios segundo o percentual de utilização do recurso repassado pelo governo federal para a compra da agricultura familiar, bem como classificar os alimentos solicitados nas chamadas públicas de 2018 conforme a extensão e propósito do processamento. Os municípios que não conseguem cumprir a legislação são aqueles que recebem mais recursos e situados em regiões urbanas. Os gêneros alimentícios mais solicitados nas chamadas públicas são in natura e/ou minimamente processados.

Palavras-chave: Alimentação Escolar; Agricultura; Segurança Alimentar e Nutricional; Política Pública; Desenvolvimento Sustentável.

1 Universidade Federal Fluminense. E-mail: diaspc2@gmail.com

2 Universidade Federal Fluminense. E-mail: kamillabertu@hotmail.com

3 Universidade Federal Fluminense. E-mail: daniele_ferreira@id.uff.br

${ }^{4}$ Organização das Nações Unidas para Alimentação e Agricultura. E-mail: luiz.dasilva@fao.org

5 Universidade Federal Fluminense. E-mail: danielebastos@id.uff.br

6 Universidade Federal Fluminense. E-mail: patihenriques@gmail.com

7 Universidade Federal Fluminense. E-mail: spereira@id.uff.br

8 Universidade Federal Fluminense. E-mail: burlandy@uol.com.br

${ }^{9}$ Universidade Federal Fluminense. E-mail: roseanesampaio@id.uff.br 
Abstract: The objective of the study was to analyze the purchasing profile of family farming for the National School Food Program in municipalities in the state of Rio de Janeiro. It sought to understand the sociodemographic characteristics of the municipalities according to the percentage of use of the resource transferred by the federal government for the purchase of family farming, as well as to classify the food ordered in the 2018 public calls according to the extent and purpose of the processing. Municipalities that fail to comply with legislation are those that receive more resources and are located in urban regions. The most requested foodstuffs in public calls are in natura and / or minimally processed.

Keywords: School Feeding; Agriculture; Food Security; Public Policy; Sustainable Development.

\section{Introdução}

O Programa Nacional de Alimentação Escolar (PNAE) é o maior e mais abrangente na área de alimentação escolar no mundo. Com a ampliação das ações de Segurança Alimentar e Nutricional (SAN) no Brasil, o PNAE se configurou como uma potente e mais duradoura política pública nesse campo (PEIXINHO, 2013).

A Lei 11.947 de 16 de junho de 2009 estabeleceu a obrigatoriedade da utilização de no mínimo $30 \%$ dos recursos financeiros repassados pelo Fundo Nacional de Desenvolvimento da Educação (FNDE) com a compra de gêneros alimentícios da agricultura familiar (AF) e do empreendedor da família rural ou de suas organizações e assim reafirmou o PNAE com uma política de SAN. A Lei determina que a prioridade da compra seja de assentados da reforma agrária, de comunidades tradicionais indígenas e comunidades quilombolas, reiterando o compromisso com a inclusão produtiva e preservação da sociobiodiversidade (BRASIL, 2009). Além disso, introduziu a Chamada Pública (CP) como um novo dispositivo jurídico com vista a facilitar o acesso de agricultores familiares ao mercado institucional público (BRASIL, 2009; PEIXINHO, 2013).

O estabelecimento de critérios de prioridade de compra levando em consideração a proximidade das unidades escolares possibilitou uma alimentação escolar mais conectada aos hábitos culturais locais, menor logística de entrega, aproximação entre agricultor e consumidor e o incentivo à sistemas alimentares mais saudáveis (CARVALHO, 2009; SARAIVA et al., 2013; SIDANER et al., 2013; TURPIN, 2009). A experiência brasileira de regulação das compras institucionais para a $A F$, inaugurada com o Programa de Aquisição de Alimentos em 2003 (BRASIL, 2003) e potencializada em 2009 por meio do PNAE, colocou o Brasil em lugar de destaque, assumindo papel de "exportador" da ideia de mercados institucionais para a agricultura familiar, inspirando outros países (GRISA et al., 2018).

Diferentes cenários podem ser observados no processo de compra da AF para o PNAE nos territórios brasileiros, desde processos bem-sucedidos ao completo descumprimento da legislação, são observadas variações entre 
regiões, estados e municípios (CASTELLANI et al., 2017; TRICHES et al., 2016). Alguns fatores vêm se destacando como desafios; o planejamento da produção, a burocracia, a logística demandada, a falta de comunicação entre os segmentos e setores envolvidos, entre outras questões (TRICHES et al., 2019).

Os municípios do Estado do Rio de Janeiro (RJ) possuem características diversas, alguns com grande vocação agrícola e outros fortemente urbanizados; apresentam perfil socioeconômico, político e institucional diferenciados que impõe desafios singulares para o delineamento de estratégias que viabilizem a compra institucional de alimentos da AF. Conhecer a realidade do Estado quanto, às características do território, dos produtores e as dificuldades para o acesso ao mercado das compras públicas contribui para o planejamento de estratégias em favor da inclusão de produtores locais ao potente mercado institucional e, simultaneamente, para ampliação da oferta de alimentos in natura na alimentação escolar. Assim, o objetivo deste estudo foi analisar o perfil de compras da agricultura familiar para o PNAE em municípios do Estado do RJ.

\section{Metodologia}

Trata-se de um estudo descritivo e transversal que buscou compreender as características sociodemográficas dos municípios $(n=92)$ do Estado do Rio de Janeiro segundo o percentual de utilização do recurso repassado para a compra de gêneros alimentícios da AF para o PNAE. Identificou-se também o tipo de alimento solicitado nas CP disponíveis em 2018.

Os dados secundários referentes ao valor do recurso repassado para os municípios do RJ e o valor utilizado para a compra de gêneros alimentícios da AF para o PNAE de 2011 a 2017 foram obtidos no sítio eletrônico do Fundo Nacional de Desenvolvimento da Educação (FNDE), na seção "Dados da Agricultura Familiar" e assim calculado o percentual de utilização do recurso repassado pelo FNDE (BRASIL, 2017). A variável percentual de utilização do recurso repassado para a compra de gêneros alimentícios da AF foi categorizada em não comprou da agricultura familiar, utilizou $<30 \%$ do recurso repassado e utilizou $30 \%$ ou mais do recurso repassado pelo governo federal.

As chamadas públicas de 2018 foram obtidas nos portais de transparência dos respectivos municípios e no sítio eletrônico do extinto Ministério do Desenvolvimento Agrário, mais especificamente na seção "Sistema de Monitoramento de Oportunidades de Compras Públicas da Agricultura Familiar" (BRASIL, 2017). Foram coletadas CP do ano de 2018, visto que era o último ano completo à época da coleta de dados.

Os municípios foram caracterizados conforme classificação regional proposta pelo Centro Estadual de Estatísticas, Pesquisa e Formação de Servidores Públicos do Rio de Janeiro (CEPERJ) em região metropolitana, costa verde, médio paraíba, centro sul fluminense, norte fluminense, noroeste 
fluminense, serrana e baixadas litorâneas (CEPERJ, 2018). A caracterização sociodemográfica considerou os dados de índice de desenvolvimento humano municipal (IDHM) e área territorial, sendo coletados no sítio eletrônico do Instituto Brasileiro de Geografia e Estatística (IBGE), na plataforma IBGE Cidades (IBGE, 2017); e a tipologia do território de cada município foi coletada no sítio eletrônico do IBGE, sendo divididos em rurais, urbanos e intermediários, podendo ser adjacente (aos centros urbanos de maior hierarquia) ou não (IBGE, 2017). A variável "total de agricultores familiares cadastrados no Programa Nacional de Fortalecimento da Agricultura Familiar (PRONAF)" foi coletada no site do IBGE (IBGE, 2017), sendo considerados para esta variável, os grupos PRONAF $\mathrm{V}$ e $\mathrm{B}$ elencados pelo IBGE que considera a agricultura familiar de acordo com o Decreto no 9.064 (2017) e PRONAF referente ao Manual de Crédito Rural/BACEN (2018). No site da Secretaria Especial de Agricultura Familiar e do Desenvolvimento Agrário (SEAD) foram coletados os números de DAP ativas em 2021, por município, sendo considerado para este dado o somatório de DAP - pessoa física com DAP - pessoa jurídica ativas no momento da consulta (SEAD, 2021).

Os gêneros alimentícios requisitados nas chamadas públicas foram contabilizados pelo número de vezes que foram solicitados e foram classificados conforme a extensão e propósito do processamento. Para classificar os alimentos considerou-se a especificação descrita no edital, sendo estes classificados como processados quando não eram in natura, minimamente processados e não apresentavam a indicação da restrição de açúcar e ou sal. Os alimentos foram classificados como ultraprocessados quando não havia na especificação do edital a indicação da restrição de aditivos (corantes, aromatizantes, conservantes, entre outros) (BRASIL, 2014; MONTEIRO et al, 2016).

Para o tratamento estatístico foi utilizado o software JASP®. Para a análise do perfil sociodemográfico dos municípios segundo categorias foi realizado, primeiramente, o teste de normalidade Shapiro-Wilk e constatou-se que a variável IDHM é paramétrica e as variáveis área territorial, valor repassado pelo FNDE, total de agricultores cadastrados no PRONAF são nãoparamétricas. Para a variável paramétrica, foi utilizado o valor médio (desvio padrão) e foi aplicado o teste $T$ student com duas categorias. Já para as variáveis não-paramétricas, foi utilizada a mediana [mínimo; máximo] e foi aplicado o teste Mann-Whitney com duas categorias.

Para comparação das médias referente ao percentual de compra da AF por região foi utilizado o teste ANOVA. Foi aplicado o teste qui-quadrado para associação entre a variável (dependente) categoria de percentual de utilização do recurso repassado para a compra da AF e o tipo de território dos municípios. 


\section{Resultados}

Participaram do estudo todos os municípios $(n=92)$ do Estado do RJ, sendo excluídos da análise um município do ano de 2012, três municípios do ano de 2013, um município do ano de 2015, dois municípios em 2016, um em 2017 e um município na variável "total de agricultores cadastrados no PRONAF" em razão de inconsistência nos dados.

A série histórica dos percentuais de utilização do recurso do governo federal com a compra de gêneros da AF para o PNAE entre os anos de 2011 a 2017 permite apontar que houve uma diminuição do percentual de municípios que não compraram da $A F$ e um aumento dos que atingiram o percentual mínimo de utilização estabelecido na Lei no 11.947 (2009), com oscilações nos dois últimos anos. Porém identifica-se uma manutenção do percentual de municípios na categoria "utiliza de $0,01 \%$ a $29,99 \%$ do recurso repassado". A comparação entre 2017 e 2016 aponta para um aumento do percentual dos municípios que "não compraram da AF" e uma redução daqueles que utilizam $30,0 \%$ ou mais do recurso repassado (Figura 1). Dos 92 municípios, 4 não compraram da AF entre 2011 e 2017 (dados não apresentados em tabela).

Os percentuais médios de utilização do recurso repassado para a compra de gêneros alimentícios da agricultura familiar vêm avançando no Estado do RJ de forma similar à média nacional, que em 2017 atingiu cerca de $22 \%$. Entre 2011 a 2017 cerca de $41,26 \%$ dos municípios permaneceram sem conseguir atender os $30 \%$ de utilização dos recursos (dados não apresentados em tabela).

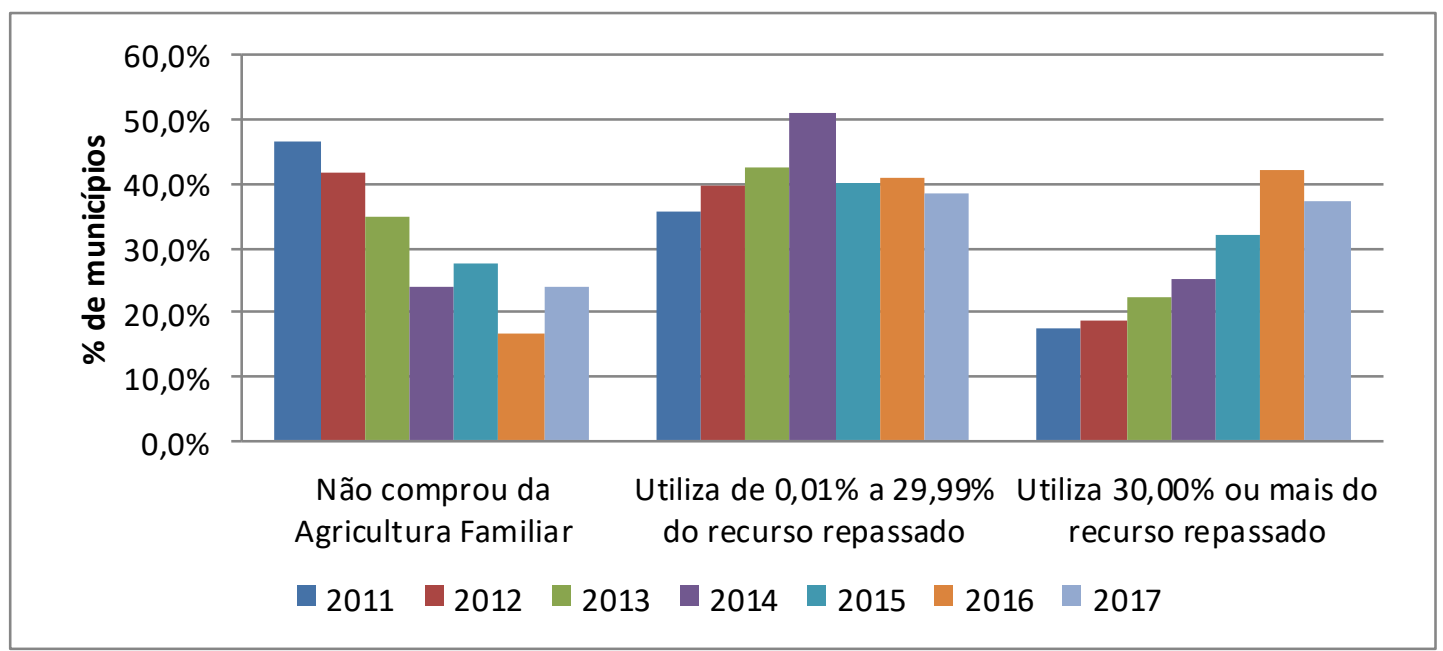

Figura 1: Distribuição do percentual de municípios do Estado do Rio de Janeiro segundo percentual de utilização do recurso repassado pelo governo federal para a compra de gêneros alimentícios da agricultura familiar para o Programa Nacional de Alimentação Escolar entre os anos de 2011 e 2017. Fonte: Brasil (2017). Organização dos autores.

A análise dos últimos dados disponíveis (2017) aponta que 57 (62,6\%) dos 91 municípios analisados, utilizaram $<30 \%$ do recurso repassado, sendo que destes, 22 (38,6\% - dados não mostrados em tabela) não utilizaram 
nenhum recurso com a compra da $\mathrm{AF}$ e $34(37,4 \%)$ atingiram o percentual de utilização de $30 \%$ ou mais do valor repassado para a compra de gêneros alimentícios da AF (Tabela 1).

Os municípios que compraram abaixo dos $30 \%$ possuem um valor médio de IDHM e mediana da área territorial maiores do que os que atingiram os $30 \%$ ou mais (Tabela 1 ).

A mediana da variável "total de agricultores cadastrados no PRONAF" foi maior entre os municípios classificados na categoria de utilização $30 \%$ ou mais em relação aos que compram abaixo desse percentual (Tabela 1).

Não houve diferença significativa das variáveis IDHM, área territorial e total de agricultores ligados ao PRONAF segundo percentual de recursos utilizado para a compra de gêneros alimentícios da AF. Houve diferença significativa na mediana do "valor repassado pelo FNDE" entre municípios que utilizaram valores abaixo de $30 \%$ e igual ou acima de $30 \%$. (Tabela 1 ).

Tabela 1: Valores médios de variáveis econômicas, sociais e demográficas associadas ao percentual de utilização do recurso repassado para a compra de gêneros alimentícios nos municípios do Rio de Janeiro no ano de 2017.

\begin{tabular}{lccc}
\hline \multirow{2}{*}{ Variáveis } & \multicolumn{2}{c}{ \% utilizado para a compra de gêneros alimentícios da agricultura } & \multirow{2}{*}{$\mathrm{P}$} \\
\cline { 2 - 3 } & \multicolumn{2}{c}{ familiar } & \\
\cline { 2 - 3 } IDHM $^{\mathrm{a}}$ & $0,70 \%(\mathrm{n}=57)$ & $030 \%(\mathrm{n}=34)$ & 0,702 \\
Valor repassado pelo & $(0,031)$ & $(0,044)$ & \multirow{2}{*}{0,03} \\
FNDE $(\mathrm{R} \$)^{\mathrm{b}}$ & $813.974,00$ & $399.412,00$ & \\
& {$[9.087,00 ; 76.700 .000,00]$} & {$[100.376,00 ; 4.040 .000,00]$} & 0,03 \\
Área Territorial $\left(\mathrm{km}^{2}\right)^{\mathrm{b}}$ & 307,39 & 405,71 & 0,12 \\
Total de agricultores & {$[19,39 ; 4.031,99]$} & {$[50,54 ; 1.118,04]$} & \\
cadastrados no & 256 & 350 & 0,24 \\
PRONAFb & {$[0 ; 4967]$} & {$[8 ; 2.869]$} & \\
\hline
\end{tabular}

Fonte: Brasil (2017); Instituto Brasileiro de Geografia e Estatística (2017); IBGE Cidades (2017). Organização dos autores.

$a$ = paramétrica - Teste $\mathrm{t}$ student - média (desvio padrão)

$\mathrm{b}=$ não-paramétrica - Teste Mann-Whitney - mediana [mínimo; máximo]

FNDE = Fundo Nacional de Desenvolvimento da Educação

IDHM= Índice de Desenvolvimento Humano Municipal

PRONAF = Programa Nacional de Fortalecimento da Agricultura Familiar

Não houve diferença significativa entre o percentual médio de recurso utilizado para a compra de gêneros alimentícios em 2017 por regiões do Estado do RJ. Contudo, a região Serrana apresentou um valor absoluto maior e a região Médio-Paraíba um valor menor em relação às demais regiões (Tabela 2). Identificou-se o número de DAP ativas por região, sendo: Serrana $n=3499$; Noroeste Fluminense $n=2486$; Norte Fluminense $n=1952$; Baixadas Litorâneas $n=934$; Metropolitana $n=900$; Médio Paraíba $n=892$; Centro Sul-Fluminense $\mathrm{n}=631$; Costa Verde $\mathrm{n}=240$ (dados não mostrados em tabelas). 
Tabela 2: Valores médios do percentual de utilização do recurso repassado para a compra de gêneros alimentícios provenientes da agricultura familiar das regiões do Estado do Rio de Janeiro no ano de 2017.

\begin{tabular}{lcccc}
\hline Região & $\begin{array}{c}\text { Percentual médio de } \\
\text { recurso utilizado com a } \\
\text { AF }\end{array}$ & \multicolumn{3}{c}{$\begin{array}{c}\text { Número } \\
\text { de } \\
\text { Municípios }\end{array}$} \\
\hline Região Serrana & $(\%)$ & Desvio Padrão & N & P \\
Região Centro Sul-Fluminense & 34.466 & 21.364 & 14 & 0,069 \\
Região Noroeste Fluminense & 31.388 & 19.382 & 10 & \\
Região Norte Fluminense & 25.942 & 21.007 & 13 & \\
Região das Baixadas Litorâneas & 24.534 & 15.997 & 8 & \\
Região Metropolitana & 19.529 & 17.882 & 12 & \\
Região Costa Verde & 16.797 & 20.614 & 19 & \\
Região Médio Paraíba & 15.757 & 13.903 & 3 & \\
\hline
\end{tabular}

Fonte: Brasil (2017); Centro Estadual de Estatísticas, Pesquisas e Formação de Servidores Públicos do Rio de Janeiro (2018). Organização dos autores.

*Teste ANOVA

Houve diferença significativa entre o percentual de municípios urbano, rural adjacente e intermediário adjacente segundo categoria de utilização do recurso federal com a aquisição de gêneros da AF. A maioria dos municípios rurais adjacentes atingem o percentual de utilização do recurso estabelecido na Lei e a maioria dos municípios urbanos não atinge (Tabela 3).

Tabela 3: Percentual de utilização do recurso repassado pelo Fundo Nacional de Desenvolvimento da Educação para a compra de gêneros alimentícios da agricultura familiar para a alimentação escolar no ano de 2017 segundo a tipologia do território dos municípios do Rio de Janeiro.

\begin{tabular}{|c|c|c|c|c|}
\hline \multirow[t]{2}{*}{ Tipo de território } & \multicolumn{2}{|c|}{$\begin{array}{c}\text { \% de utilização do recurso repassado para a } \\
\text { compra de gêneros alimentícios da } \\
\text { agricultura familiar }\end{array}$} & \multirow[t]{2}{*}{ Total } & \multirow[t]{2}{*}{$\mathrm{P}$} \\
\hline & $\begin{array}{c}<30 \% \\
\mathrm{n}(\%) \\
\end{array}$ & $\begin{array}{c}\geq 30 \% \\
\mathrm{n}(\%)\end{array}$ & & \\
\hline Rural Adjacente & $6(32 \%)$ & $13(68 \%)$ & $19(100 \%)$ & \\
\hline Urbano & $45(74 \%)$ & $16(26 \%)$ & $61(100 \%)$ & 0.003 \\
\hline Intermediário Adjacente & $6(54 \%)$ & $5(46 \%)$ & $11(100 \%)$ & \\
\hline Total & 57 & 34 & 91 & \\
\hline
\end{tabular}

Fonte: Brasil (2017) e Instituto Brasileiro de Geografia e Estatística (2017). Organização dos autores.

*Teste qui-quadrado

Foram localizados 49 editais de chamadas públicas de 40 municípios publicadas no ano de 2018; sendo 8 da região Serrana, 6 da região Centro Sul Fluminense, 6 da região Norte Fluminense, 6 da região Médio Paraíba, 5 da região Noroeste Fluminense, 5 da região Metropolitana, 2 da região Costa Verde, 2 da região Baixadas Litorâneas.

Revbea, São Paulo, V. 16, № 6: 360-377, 2021. 
A análise das CP permitiu identificar 1031 alimentos solicitados, a maioria dos gêneros alimentícios solicitados foram classificados como alimentos in natura e ou minimamente processados e em pequena quantidade, alimentos classificados como ultraprocessados (Tabela 4).

Tabela 4: Classificação segundo a extensão e propósito de processamento dos gêneros alimentícios solicitados em chamadas públicas dos municípios do Rio de Janeiro no ano de 2018.

\begin{tabular}{lccc}
\hline Classificação & $\begin{array}{c}\text { No de vezes que } \\
\text { o item foi } \\
\text { solicitado }\end{array}$ & $\%$ & Gêneros alimentícios \\
\hline $\begin{array}{l}\text { Alimentos in natura e } \\
\text { minimamente } \\
\text { processados }\end{array}$ & 996 & 96,6 & $\begin{array}{c}\text { Frutas, hortaliças, leguminosas, cereais, } \\
\text { polpa de frutas, entre outros. }\end{array}$ \\
$\begin{array}{l}\text { Alimentos processados } \\
\text { Alimentos } \\
\text { ultraprocessados }\end{array}$ & 14 & 1,4 & $\begin{array}{c}\text { Queijo minas, picolé de frutas, beiju, biscoito } \\
\text { de polvilho, entre outros. }\end{array}$ \\
\hline \multicolumn{1}{c}{ Total } & 21 & $\begin{array}{c}\text { logurte, bebida láctea, requeijão, bebida } \\
\text { mista de juçara, entre outros. }\end{array}$ \\
\hline $\begin{array}{l}\text { Fonte: Brasil (2017); Editais de chamadas públicas dos municípios do Rio de Janeiro. Organização dos } \\
\text { autores. }\end{array}$
\end{tabular}

\section{Discussão}

A análise da evolução da compra da AF para o PNAE aponta para um crescimento deste mercado institucional no Estado do RJ. No entanto, existe um número considerável de municípios que não compram ou não conseguem atingir o percentual estabelecido em Lei desde 2011, o que repercute em uma média dos municípios do Estado abaixo dos 30\% (BRASIL, 2009; BRASIL, 2017). Quatro municípios não utilizaram o recurso para aquisição de gêneros da AF entre 2011 a 2017, destes 3 são urbanos e 1 intermediário adjacente (IBGE, 2017; BRASIL, 2017). Em 2017, a maior parte dos municípios utilizou algum recurso para aquisição de gêneros da $A F$, mas não conseguiu atingir o percentual mínimo previsto na legislação, o que sugere avanços, mas também desafios na execução dessa importante política pública. Os dados não permitem verificar se a compra ocorreu de forma mais local, se os gêneros alimentícios são orgânicos/agroecológicos e/ou adquiridos de assentados da reforma agrária, comunidades tradicionais indígenas e comunidades quilombolas; tais constatações são importantes para verificar o potencial de inclusão produtiva desses segmentos no mercado institucional do PNAE (WAGNER; GLEHLEN, 2015).

A dificuldade de grande parte dos municípios atingir os $30 \%$ tem se mostrado persistente ao longo dos anos. Segundo estudos, os principais fatores relacionados à estas dificuldades são: a inviabilidade de fornecimento regular de gêneros, impossibilidade de emissão de nota fiscal por parte dos agricultores, inadequação à legislação sanitária, obtenção da Declaração de Aptidão ao PRONAF (DAP), logística de entrega, divulgação insuficiente das 
chamadas públicas, falta de organização dos agricultores, pouca articulação entre gestores e agricultores (FERREIRA et al., 2019; MONEGO, 2014; SARAIVA et al., 2013; TEO et al., 2017).

Ainda que as diferenças entre os indicadores sociodemográficos não tenham se mostrado significativas, percebe-se que as médias do IDHM são consideradas altas nos municípios do Estado (IPEA, 2013), sendo discretamente mais elevada nos municípios que não compram da AF. Dias et al (2020) encontraram relação inversa entre IDH das capitais brasileiras e percentual de utilização de recursos com a compra da AF e sugere que municípios mais urbanizados e supostamente mais desenvolvidos podem ter mais dificuldade no cumprimento da legislação. Segundo os autores as capitais que possuem um menor quantitativo de alunos, e por consequência recebem valores absolutos mais baixos de recurso do governo federal, estão situados entre aquelas que atingem o percentual mínimo estabelecido pela legislação (DIAS et al., 2020). Triches et al. (2019) verificaram melhor aproveitamento dos recursos do programa em municípios de médio e pequeno porte, e relacionaram aos melhores níveis de participação, diálogo e parcerias entre gestores, agricultores, conselheiros e extensão rural.

Os municípios que recebem maior recurso possuem maior número de escolas e, portanto, maior demanda de gêneros alimentícios, exigindo assim maior capacidade de produção e organização dos agricultores para atender a uma logística de entrega mais complexa (DIAS et al., 2020; MACHADO et al., 2018; OLIVEIRA et al., 2018). Ademais, as possíveis resistências na adoção de novas práticas institucionais, acirrada por conflitos de interesse no âmbito da gestão das compras públicas pode refletir em inércia dos governos locais e facilitar a manutenção dos processos tradicionais de licitação em detrimento da $\mathrm{CP}$, ou mesmo realizá-la de forma irregular, sem respeitar os critérios definidos em legislação, tais como garantir o levantamento da produção local, realizar adequada pesquisa e definição de preços, incluir lista de substituição de gêneros, realizar ampla divulgação, entre outros, e assim dificultar o processo de aquisição de gêneros da AF (BRASIL, 2020; DIAS et al., 2020; THE LANCET, 2019; TRICHES; SILVESTRI, 2018).

Observou-se que os grandes centros urbanos possuem maior dificuldade de realizar a compra de gêneros alimentícios da AF, como é o caso do Rio de Janeiro e Duque de Caxias que, em 2017, receberam os dois maiores repasses do governo federal e respectivamente um utilizou menos de $1 \%$ do recurso e o outro não comprou da AF. Resultado semelhante foi encontrado por Santos e Evangelista (2016) que identificaram que os grandes centros urbanos de Minas Gerais que recebiam maior quantia de recurso apresentaram maior dificuldade em realizar a compra da AF. Os autores associaram a dificuldades na logística de entrega, inadequação dos locais de armazenamento, pequena extensão territorial para a produção agrícola, grau elevado de urbanização, pouca disponibilidade de agricultores familiares, entre outras (SANTOS; EVANGELISTA, 2016). Esses achados vêm sendo reiterados 
em outros estudos (DIAS et al., 2020; MACHADO et al., 2018; MONEGO, 2014).

O número absoluto de agricultores cadastrados no PRONAF e consequentemente possuidores de Declaração de Aptidão ao PRONAF (DAP) foi maior nos municípios que atingiram os $30 \%$ de utilização do recurso do FNDE com a AF. Destaca-se que existem agricultores familiares possuidores de DAP não cadastrados no PRONAF que podem acessar a política pública. $A$ DAP é o documento que identifica e qualifica os agricultores familiares e suas organizações, para o acesso às políticas públicas voltadas para esse segmento (BRASIL, 2018) e vem sendo mencionado como determinante para o sucesso da compra de gêneros da AF (MONEGO, 2014; OLIVEIRA et al., 2018; SANTOS; EVANGELISTA, 2016).

O processo de aquisição da DAP de modo geral está relacionado com o acesso dos agricultores à assistência técnica. Guazinroli \& Vinchon (2019) identificaram que a assistência técnica influenciou positivamente na renda bruta dos agricultores familiares nas regiões Serrana, Norte e Noroeste do RJ. Oliveira et al (2018) verificaram que a maioria (92,9\%) dos agricultores familiares de municípios goianos que vendem para o PNAE tiveram acesso à Assistência Técnica e Extensão Rural (ATER). A falta de investimento em políticas públicas voltadas para o agricultor familiar, em razão dos inúmeros cortes orçamentários e da escassez de recursos humanos nas instituições de assistência técnica reduz a capacidade de atendimento nos estados e municípios, compromete especialmente o pequeno agricultor que não possui renda para investir em uma assistência técnica privada (CASTRO, 2015; LANDINI, 2015).

O presente estudo constatou que os municípios caracterizados como urbanos em sua maioria não atingem o percentual mínimo de compra da AF, diferentemente dos rurais. A região serrana obteve melhor desempenho em relação ao cumprimento da legislação, sendo a região que possui um quantitativo maior de municípios rurais adjacentes e o maior número de DAP ativas (BRASIL, 2017; IBGE, 2017). Apesar da presença da indústria têxtil e do ecoturismo, a região possui uma potente produção agrícola oriunda dos agricultores familiares, com forte cultivo de hortifrutigranjeiros e produção de orgânicos e hidropônicos (CEPERJ, [2010-2020]; MARAFON, 2017). As áreas rurais fluminenses apesar de apresentarem nuances de urbanização e 0 fenômeno da pluriatividade econômica, ainda conseguem manter as atividades agrícolas (MARAFON, 2017; SOUZA, 2019).

A região do Médio Paraíba é integrada por 9 municípios urbanos, 2 rurais adjacentes e 1 intermediário adjacente e obteve 0 menor percentual médio de compra (BRASIL, 2017; CEPERJ, [2010-2020]; IBGE, 2017). Segundo a CEPERJ ([2010-2020]) é a segunda região mais industrializada do Rio de Janeiro com presença de grandes indústrias; porém possui uma forte produção de leite (CEPERJ, [2010-2020]; MARAFON, 2017). A melhora no perfil de compra poderia ser alcançada com o mapeamento dos agricultores e 
da produção local, a articulação entre atores locais, especialmente as ATER, Vigilância Sanitária, representantes do Ministério da Agricultura, Pecuária e Abastecimento (MONEGO, 2014; RODRIGUES et al., 2017). Nas CP encontradas de dois municípios da região do Médio Paraíba verificou-se que foram solicitados iogurte, manteiga e leite pasteurizado, alimentos produzidos localmente que poderiam ser fornecidos para outros municípios da região.

Os municípios das regiões predominantemente urbanas, embora tenham mais dificuldade com a compra local, podem equacionar esse desafio por meio da articulação mais regional e aproveitar o potencial da região intermediária que circunscreve o município (BRASIL, 2016). A compra local diminui o custo com a logística e a dependência dos atravessadores; além da manutenção dos agricultores em atividades agrícolas. Segundo Guanziroli e Vinchon (2019) as atividades não-agrícolas não estão relacionadas com aumento na renda bruta dos agricultores. Além disso, os agricultores familiares, por vezes, dependem dos atravessadores para obter renda advinda da sua produção, reduzindo assim a remuneração pela sua produção; desestimulando a permanência nas atividades agrícolas (MARAFON, 2017; RODRIGUES et al., 2017). A valorização de cadeias curtas é a garantia da soberania alimentar dos povos, com formas de cultivo mais sustentáveis e respeito à cultura alimentar dos escolares; e o processo de inclusão produtiva dos segmentos de comunidades tradicionais que pode favorecer a compra de gêneros orgânicos e agroecológicos (CARVALHO, 2009; MACHADO et al., 2018; MONEGO, 2014).

As CP são instrumentos jurídicos fundamentais para o estimulo aos circuitos curtos de produção e consumo e trazem ganhos para a composição dos cardápios, privilegiando alimentos in natura e minimamente processados produzidos pela AF. Esse processo é facilitado em razão da CP não prever a disputa de preços como critério, diferentemente da licitação que historicamente favorece grandes empresas (BACCARIN et al., 2017).

Nesse estudo verificou-se que as CP priorizaram a solicitação de alimentos in natura e minimamente processados, em conformidade com o Guia alimentar para a população brasileira (BRASIL, 2014), e já converge com as novas diretrizes alimentares propostas na resolução no6 (2020) do FNDE para o PNAE (BRASIL, 2020; CARVALHO, 2009; MONTEIRO et al., 2016). Estudos apontam a necessidade de aumentar a quantidade de vegetais, frutas e legumes e diminuir a de ultraprocessados nos cardápios de municípios brasileiros (BOITO et al., 2019; CAMARGO et al., 2019; GABRIEL et al., 2012). Assim, a compra da agricultura familiar pode oportunizar um maior consumo de alimentos in natura e minimamente processados pelos alunos na alimentação escolar se os gestores estiverem atentos à produção agrícola local (BACCARIN et al., 2017; CARMAGO et al., 2019; FUHR; TRICHES, 2017).

O conteúdo da CP pode facilitar ou dificultar o acesso dos agricultores familiares ao mercado institucional, dependendo da sazonalidade, da escolha dos alimentos, da forma como são especificados e da previsão de critérios para substituição de gêneros (BRASIL, 2016; FUHR; TRICHES, 2017; MONEGO, 
2014; SANTOS; EVANGELISTA, 2016; DIAS et al., 2020). A divulgação e publicização parece restrita, visto que foram encontradas chamadas públicas de apenas 43,5\% dos municípios, sendo que em 2017 cerca de 75\% dos municípios realizaram a compra de gêneros da AF.

Segundo o IBGE (2011) o Rio de Janeiro é o Estado com o maior grau de urbanização do Brasil $(96,7 \%)$ e no espaço fluminense há uma extrapolação da urbanização para as áreas rurais trazendo novas atividades econômicas para o campo. As profissões consideradas mais urbanas começam a ser oferecidas em municípios rurais fluminenses, o que leva os agricultores a alternarem entre atividades agrícolas e não agrícolas com o objetivo de complementação da sua renda (GUANZIROLI; VINCHON, 2019). Porém, verifica-se no presente estudo que os municípios rurais adjacentes são os que mais alcançam o percentual mínimo instituído por Lei (BRASIL, 2009), indicando que o PNAE se mostra com uma oportunidade para os agricultores familiares melhorarem a sua renda e se manterem em atividades agrícolas (GUANZIROLI; VINCHON, 2019; MARAFON, 2017; SOUZA, 2019).

No contexto de elevado grau de urbanização, a agricultura familiar urbana (AFU) representa uma alternativa capaz de potencializar as compras públicas nesses territórios, proporcionando uma conciliação entre geração de renda com a conservação da biodiversidade, ao mesmo tempo em que promovem a recuperação de florestas, a preservação de tradições culturais e a produção de alimentos nutritivos e saborosos, promovendo a segurança alimentar e nutricional (RAMOS, 2019; MACHADO; MACHADO, 2002; AQUINO; ASSIS, 2017; LINHARES et al., 2018). O fortalecimento da AFU exige políticas públicas específicas voltadas para essa atividade e para esse segmento produtivo. Os espaços urbanos precisam ser (re)valorizados como áreas destinadas não somente para produção de alimentos para autoconsumo, mas para a comercialização em feiras e mercados institucionais públicos, principalmente em municípios que não têm área rural, estimulando cadeias curtas de produção e a economia local. $\mathrm{O}$ apoio do poder público juntamente com uma sociedade organizada é estratégico para a promoção da AFU. Em 2003, o governo federal por meio do Ministério do Desenvolvimento Social e Combate à fome incentivou a implantação de hortas comunitárias em espaços urbanos, que tornaria acessível os alimentos saudáveis aos moradores do entorno. Outra ação importante para o fortalecimento da agricultura urbana foi a iniciativa Circuito Carioca de Feiras Orgânicas (resultado da mobilização de grupos relacionados à agricultura familiar e agroecologia) mais uma opção de mercado institucional para os agricultores (FERNANDEZ; FILHO, 2019).

Souza (2019) destaca que o espaço agrário fluminense necessita de políticas públicas mais especializadas, ou seja, mais conectadas com as potencialidades e desafios do contexto. $O$ arcabouço legal do PNAE oportuniza a aproximação com contexto local e, portanto, demanda soluções singulares para cada realidade. É preciso compreender os desafios do território fluminense em diálogo com as políticas públicas locais para o adequado investimento em soluções que facilitem o acesso dos agricultores familiares

revista brasileira educação ambiental 
fluminenses ao mercado institucional do PNAE (CARVALHO, 2009; MONEGO, 2014; RODRIGUES, 2017).

\section{Conclusão}

Em síntese, a compra de gêneros alimentícios vem avançando nos municípios do Estado RJ, mas ainda se encontra aquém do desejável especialmente em regiões mais urbanizadas e que recebem maior volume de recursos. Evidencia-se que a oferta de alimentos mais saudáveis na alimentação escolar vem avançando, especialmente naqueles municípios que executam as CP, tendo em vista o perfil de gêneros alimentícios solicitados. A promoção de uma alimentação adequada e saudável também se baseia no estímulo de um sistema alimentar social e ambientalmente sustentável.

O estudo de forma inédita contribui com a caracterização das compras públicas da AF para o PNAE dos municípios do RJ de modo a compreender como as características sociodemográficas, territoriais e acesso aos recursos interagem nesse processo. Destacam-se os limites de estudos baseados em dados secundários, que não oportunizam compreender as dinâmicas de interação entre atores e instituições envolvidas com as compras públicas da AF para PNAE, sugerindo, portanto, uma agenda de pesquisa necessária à compreensão das especificidades que caracterizam a relação entre os diferentes atores locais no território fluminense.

Agradecimentos: $O$ presente trabalho foi realizado com apoio da Coordenação de Aperfeiçoamento de Pessoal de Nível Superior - Brasil (CAPES) - Código de Financiamento 001, do Conselho Nacional de Desenvolvimento Científico e Tecnológico (CNPq) e da Pró-Reitoria de Pesquisa, Pós-Graduação e Inovação da Universidade Federal Fluminense.

\section{Referências}

AQUINO, A. M.; ASSIS, R. L. Agricultura orgânica em áreas urbanas e periurbanas com base na agroecologia. Ambiente \& Sociedade, Campinas, v.10, n.1, p.137-150, jun 2017.

BACCARIN, J. G.; TRICHES, R. M.; TEO, C. R. P. A; SILVA D. B. P. Indicadores de avaliação das compras da agricultura familiar para alimentação escolar no Paraná, Santa Catarina e São Paulo. Revista de Economia e Sociologia Rural, Brasília, v.55, n.1, p.103-122, jan/mar 2017.

BANCO CENTRAL DO BRASIL. Manual de Crédito Rural. [S.I.], 2018. Disponível em: <https://www3.bcb.gov.br/mcr>. Acesso em: 11 mai. 2020.

BOITO, T.; HARTMANN, V.; KÜMPEL, D. A.; CARLI, G. Avaliação de cardápios de uma escola de educação infantil. Revista Contexto \& Saúde, v.19, n.36, p.14-19, 2019.

Revbea, São Paulo, V. 16, № 6: 360-377, 2021. 
BRASIL, Ministério da Educação. Fundo Nacional de Desenvolvimento da Educação. Dados da agricultura familiar. Brasília, 2017. Disponível em: $<$ https://www.fnde.gov.br/index.php/programas/pnae/pnae-consultas/pnaedados-da-agricultura-familiar>. Acesso em: 19 jun. 2020.

BRASIL. Decreto no 9.064, de 31 de maio de 2017. Dispõe sobre a Unidade Familiar de Produção Agrária, institui o Cadastro Nacional da Agricultura Familiar e regulamenta a Lei no 11.326, de 24 de julho de 2006 , que estabelece as diretrizes para a formulação da Política Nacional da Agricultura Familiar e empreendimentos familiares rurais. Diário Oficial da União, Brasília, edição extra, p.11, 31 de maio de 2017. Disponível em: $<$ http://www.planalto.gov.br/ccivil 03/ ato20152018/2017/decreto/d9064.htm\#: :text=D9064\&text=Disp\%C3\%B5e\%20sobre\% 20a\%20Unidade\%20Familiar,Familiar\%20e\%20empreendimentos\%20familiare s\%20rurais>. Acesso em: 11 mai. 2020

BRASIL. Lei no 10.696, de 2 de julho de 2003. Dispõe sobre a repactuação e o alongamento de dívidas oriundas de operações de crédito rural, e dá outras providências. Diário Oficial da União, Brasília, seção 01, p.1, 3 de julho de $2003 . \quad$ Disponível em: $<$ http://www.planalto.gov.br/ccivil 03/leis/2003/L10.696.htm>. Acesso em 19 nov. 2020.

BRASIL. Lei no 11.947, de 16 de junho de 2009. Dispõe sobre o atendimento da alimentação escolar e do Programa Dinheiro Direto na Escola aos alunos da educação básica; altera as Leis no 10.880, de 9 de junho de 2004, 11.273, de 6 de fevereiro de 2006, 11.507, de 20 de julho de 2007; revoga dispositivos da Medida Provisória oㅡ 2.178-36, de 24 de agosto de 2001, e a Lei oㅡ 8.913, de 12 de julho de 1994; e dá outras providências. Diário Oficial da União, Brasília, seção 01, p.2-4, 17 junho de 2009. Disponível: <http://www.planalto.gov.br/ccivil 03/ ato2007-2010/2009/lei/l11947.htm>.

Acesso em: 19 nov. 2020.

BRASIL. Manual de Aquisição de Produtos da Agricultura Familiar para a Alimentação Escolar. Brasília, DF: Fundo Nacional de Desenvolvimento da Educação, 2016.

BRASIL. Portaria no 434, de 19 de julho de 2017. Diário Oficial da União, Brasília, seção 01, p.12, 20 de julho de 2017. Disponível em: $<$ https://www.in.gov.br/materia/-

lasset publisher/Kuirw0TZC2Mb/content/id/19186608/do1-2017-07-20-portarian-434-de-19-de-julho-de-2017-19186505>. Acesso em 19 jun. 2020.

BRASIL. Portaria no 523, de 24 de agosto de 2018. Disciplina a emissão de declaração de Aptidão ao Pronaf (DAP). Diário Oficial da União, Brasília, seção 01, p.4-6, 27 de agosto de 2018. Disponível em: $<$ https://pesquisa.in.gov.br/imprensa/isp/visualiza/index.jsp?data=27/08/2018\&j ornal=515\&pagina $=4>$. Acesso em 11 mai. 2020. 
BRASIL. Resolução no 6, de 8 de maio de 2020. Dispõe sobre o atendimento da alimentação escolar aos alunos da educação básica no âmbito do Programa Nacional de Alimentação Escolar - PNAE. Diário Oficial da União, Brasília, seção 01, p.38-44, 12 de maio de 2020. Disponível em: <https://www.in.gov.br/en/web/dou/-/resolucao-n-6-de-8-de-maio-de-2020256309972>. Acesso em 11 mai. 2020.

BRASIL. Secretaria de Atenção à saúde. Departamento de Atenção Básica. Guia alimentar para a população brasileira. Brasília, DF: Ministério da Saúde, 2014. $2^{\underline{a}}$ ed.

CAMARGO, R. G. M.; CAIVANO, S.; DOMENE, S. M. A. Avaliação qualitativa de cardápios escolares oferecidos em municípios brasileiros. Cienc Saude Colet, Rio de Janeiro, ago 2019.

CARVALHO, D. G. Licitações sustentáveis, alimentação escolar e desenvolvimento regional: uma discussão sobre o poder de compra governamental a favor da sustentabilidade. Planejamento e políticas públicas, n.32, p.115-148, jan/jun 2009.

CASTELLANI, A.L.A; TRENTINI T.; NISHIDA, W.; ROSSI, C. E.; COSTA, L. C. F.; VASCONCELOS, F. A. G. Aquisição de alimentos da agricultura familiar e orgânicos pelo Programa Nacional de Alimentação Escolar no Estado de Santa Catarina. Rev Nutr., Campinas, v.30, n.5, p.651-662, set/out 2017.

CASTRO, C.N. Desafios da agricultura familiar: o caso da assistência técnica e extensão rural. Boletim regional, urbano e ambiental, n. 12, jun/dez 2015.

CENTRO ESTADUAL DE ESTATÍSTICAS, PESQUISAS E FORMAÇÃO DE SERVIDORES PÚBLICOS DO RIO DE JANEIRO. Tabela da Divisão municipal e regional fluminense - 2018. Rio de Janeiro, 2018. Disponível em; <http://www.ceperi.ri.gov.br/Conteudo.asp?ident=79.. Acesso em: 27 jul. 2020.

CENTRO ESTADUAL DE ESTATÍSTICAS, PESQUISAS E FORMAÇÃO DE SERVIDORES PÚBLICOS DO RIO DE JANEIRO. Regiões do Estado do Rio de Janeiro. Rio de Janeiro, 2010-2020. Disponível em: $<$ http://www.ceperj.ri.gov.br/Conteudo.asp?ident=81>. Acesso em; 11 mai. 2020.

DIAS, P. C.; BARBOSA, I. R. O.; BARBOSA, R. M. S.; FERREIRA, D. M.; SOARES, K. C. B.; SOARES, D. S. B.; HENRIQUES, P.; BURLANDY, L. Compra da agricultura familiar para alimentação escolar nas capitais brasileiras. Rev Saude Publica, São Paulo, v.54, n.73, 2020.

FERNANDEZ, A.C.; FILHO, A.C.B. Agricultura familiar urbana. Cidades, n.39, 2019.

FERREIRA, D.M.; BARBOSA, R.M.S.; FINIZOLA N.C.; SOARES, D. S. B.; HENRIQUES, P.; PEREIRA, S.; PEREIRA, S.; CARVALHOSA, C.S.; SIQUEIRA, A. B. F. S.; DIAS, P. C. Percepção de agentes operadores do Programa Nacional de Alimentação Escolar. Rev Saude Publica, São Paulo, v.53, 34, 2019. 
FUHR, A. L.; TRICHES, R. M. Qualidade da alimentação escolar a partir da aquisição de produtos da agricultura familiar. Segurança Alimentar e Nutricional, Campinas, v.24, n.2, p.113-124, 2017.

GABRIEL, C. G.; COSTA, L. C. F.; CALVO, M. C. M.; VASCONCELOS, F. A. G. Planejamento de cardápios para escolas públicas municipais: reflexão e ilustração desse processo em duas capitais brasileiras. Rev Nutr., Campinas, v. 25, n.3, p.363-72, 2012.

GRISA, C.; PERAFÁN, M. E. V; CALDERÓN, P. E. G. Transferência e tradução de políticas públicas do Brasil para a Colômbia: o caso das compras públicas da agricultura familiar. Estudos Sociedade e Agricultura, Rio de Janeiro, v. 26, n.2, p.353-375, 2018.

GUANZIROLI, C. E.; VINCHON, K. Agricultura familiar nas regiões serrana, norte e noroeste fluminense: determinantes do processo de geração de renda. Revista de Economia e Sociologia Rural, Brasília, v.57, n.3, p.353-367, jul/set 2019.

INSTITUTO BRASILEIRO DE GEOGRAFIA E ESTATÍSTICA. Censo Agropecuário 2017: Tabela 6771 - Número de estabelecimentos agropecuários e Área dos estabelecimentos agropecuários, por tipologia, condição legal do produtor, existência de CNPJ, produtor possui DAP (Declaração de Aptidão ao PRONAF) e direção dos trabalhos do estabelecimento agropecuário. [S.I.], 2017. Disponível em: <https://sidra.ibge.gov.br/tabela/6771>. Acesso em 11 mai. 2020.

INSTITUTO BRASILEIRO DE GEOGRAFIA E ESTATÍSTICA. Classificação e Caracterização dos Espaços Rurais e Urbanos do Brasil. [S.I.], 2017. Disponível em: <https://www.ibge.gov.br/geociencias/organizacao-doterritorio/15790-classificacao-e-caracterizacao-dos-espacos-rurais-e-urbanosdo-brasil.html?=\&t=acesso-ao-produto $>$. Acesso em: 23 jul. 2020.

INSTITUTO BRASILEIRO DE GEOGRAFIA E ESTATÍSTICA. IBGE Cidades. [S.I.], 2017. Disponível em: <https://cidades.ibge.gov.br/>. Acesso em 11 mai. 2020.

INSTITUTO DE GEOGRAFIA E ESTATÍSTICA. Urbanização. In: INSTITUTO DE GEOGRAFIA E ESTATÍSTICA. Censo demográfico 2010. [Rio de Janeiro, RJ]: $\quad$ IBGE, $2011 . \quad$ Disponível em:

$<$ https://biblioteca.ibge.gov.br/index.php/bibliotecacatalogo?view=detalhes\&id=249230 $>$. Acesso em 11 mai. 2020.

INSTITUTO DE PESQUISA ECONÔMICA APLICADA. Índice de Desenvolvimento Humano Municipal Brasileiro. Brasília, DF: PNUD, 2013.

LANDINI, F.P. Problemas enfrentados por extensionistas rurais brasileiros e sua relação com suas concepções de extensão rural. Ciência Rural, Santa Maria, v.45, n.2, p.371-377, fev. 2015. 
LINHARES, J.F.P.; ROJAS, M.O.A.I.; RODRIGUES, M.I.A. Agricultura urbana como prática alternativa para Educação Ambiental: proposta de sistematização e ações em São Luís (MA). Revista Brasileira de Educação Ambiental, v.13, n.1, p.10-21, 2018.

MACHADO, A. T.; MACHADO, C. T. T. Agricultura urbana. Planaltina: Embrapa, 2002. (Documentos, 48).

MACHADO, P. M. O.; SCHMITZ, B. A. S.; GONZÁLEZ-CHICA, D. A.; CORSO, A. C. T.; VASCONCELLOS, F. A. G.; GABRIEL, C. G. Compra de alimentos da agricultura familiar pelo Programa Nacional de Alimentação Escolar (PNAE): estudo transversal com o universo de municípios brasileiros. Cienc Saude Colet, Rio de Janeiro, v.23, n.12, p.4153-4164, dec 2018.

MARAFON, G. J. Transformações no espaço rural fluminense: o papel da agricultura familiar e das atividades turísticas. In: MARAFON, G. J.; RIBEIRO, M. A. (orgs). Revisitando o território fluminense. [Rio de Janeiro, RJ]: EDUERJ, 2017. Disponível em: <http://books.scielo.org/id/3zhhw/07>. Acesso em 11 mai. 2020.

MONEGO, E.T. Segurança alimentar e nutricional: colocando em evidência a agricultura familiar. Demetra: Alimentação, Nutrição \& Saúde, v.9, n.2, p.431434, 2014.

MONTEIRO, C. A.; CANNON, G.; LEVY, R.; MOUBARAC, J. C.; JAIME, P.; MARTINS, A. P.; CANELLA, D.; LOUZADA, M.; PARRA, D. NOVA. A estrela brilha [Classificação dos Alimentos. Saúde Pública ]. World Nutrition, v.7, n.13, p.28-40, 2016.

OLIVEIRA, F.; MEDINA, G.; FIGUEIREDO R. Características dos agricultores que acessam o Programa Nacional de Alimentação Escolar. Revista de Política Agrícola, n.3, ano XXVII, jul/ago/set 2018.

PEIXINHO, A. M. L. A trajetória do Programa Nacional de Alimentação Escolar no período de 2003-2010: relato do gestor nacional. Cien Saude Colet, Rio de Janeiro, v.18, n.4, p. 909-916, apr/2013.

RAMOS, M. O. Boas práticas no processamento de alimentos da sociobiodiversidade. Maquiné, RS: Coletivo Catarse Editora; 2019. p.72.

RODRIGUES, R.; SIQUEIRA, H. M.; BIANCARDI, C. C. S.; ANDRADE, M. A. N.; VALENTE, L. M.; PAULA L. B. A aquisição de alimentos da agricultura familiar pelo PNAE no município de Alegre-ES. Demetra: Alimentação, Nutrição \& Saúde, v.12, n.1, p.91-112, 2017.

SANTOS, F. D.; EVANGELISTA, A. L. Reflexões em torno do perfil dos empreendimentos da agricultura familiar de Minas Gerais e sua relação com as políticas de acesso a mercados. Revista de Desenvolvimento Social, v.19, n.1, p.3-49, 8 abr 2016. 
SARAIVA, E. B.; SILVA A. P. F.; SOUSA, A. A.; CERQUEIRA, G.F.; CHAGAS, C. M. S.; TORAL, N. Panorama da compra de alimentos da agricultura familiar para o Programa Nacional de Alimentação Escolar. Cienc e Saude Colet, Rio de Janeiro, v.18, n.4, p.927-935, 2013.

SECRETARIA ESPECIAL DE AGRICULTURA FAMILIAR E DESENVOLVIMENTO AGRÁRIO. Extrato DAP. [S.I.], 2021?. Disponível em: <http://smap14.mda.gov.br/extratodap/>. Acesso em: 14 mar. 2021.

SIDANER, E.; BALABAN, D.; BURLANDY, L. The Brazilian school feeding programme: an example of an integrated programme in support of food and nutrition security. Public Health Nutr, v.16, n.6, p.989-994, 2013.

SOUZA, R. P. O desenvolvimento rural no Estado do Rio de Janeiro a partir de uma análise multidimensional. Revista de Economia e Sociologia Rural, Brasília, v.57, n.1, jan/mar 2019.

TEO, C. R. P. A.; MOSSMANN, M. P.; TAGLIETTI, R.L. Desafios e mecanismos de enfrentamento na relação entre agricultura familiar e alimentação escolar. Revista Griffos, n.43, 2017.

THE LANCET. A sindemia global da obesidade, desnutrição e mudanças climáticas. [S.I.]: Comissão The Lancet, jan. 2019.

TRICHES, R. M.; SILVESTRI F. Adequação das chamadas públicas para aquisição de produtos da agricultura familiar para a alimentação escolar. Desenvolvimento em questão, v.16, n.44, p.233-259, 2018.

TRICHES, R.M.; BARBOSA L. P.; SILVESTRI F. Agricultura familiar e alimentação escolar no Estado do Paraná: uma análise das chamadas públicas. Rev Paran Desenv, Curitiba, v.37, n.130, p.29-43, jan/jun 2016.

TRICHES, R.M.; SIMONETTI, M.G.; PEREZ, J.C.; BACCARIN J. G.; TEO, C. R. P. A. Condicionantes e limitantes na aquisição de produtos da agricultura familiar pelo Programa de Alimentação Escolar no Estado do Paraná. Redes, Santa Cruz do Sul, v.24, n.1, p.118-137, jan 2019.

TURPIN, M. E. A alimentação escolar como fator de desenvolvimento local por meio do apoio aos agricultores familiares. Segurança Alimentar e Nutricional, Campinas, v.16, n.2, p.20-42, 2009.

WAGNER, D.; GLEHLEN, I. A inserção da agricultura familiar no mercado institucional: entre o direito e o acesso ao recurso da política pública. Tempo da Ciência, Paraná, v.22, n.43, p.43-54, 2015. 